\title{
Applications of control engineering in industry 4.0: utilizing internet of things to design an agent based control architecture for smart material handling system
}

\begin{abstract}
The influence of global economy has led to changes in the conventional approaches for manufacturing companies. In this case, manufacturing companies has taken under consideration several essential characteristics such as real-time reaction to changes, quick and quality response in satisfying customer requests, in both hardware equipment and software modules by which the production processes are improved for next generation manufacturing systems. Nowadays, material handling system for manufacturing purposes is the key component for any modern manufacturing processes. Additionally, due to high variety of products and shorter response times in today's manufacturing industry, the demand for smart material handling system has increased. Therefore, to recover these demands, custom-built systems have to be implemented which requires individually created control software which deals with flow control, product routings, layout and products distribution. Internet of Things (IoT) is a concept that is set to enhance manufacturing by improving output quality and workflow efficiency. Nowadays manufacturing industries are utilizing internet of things concept as a network that connects multiple sensors and devices through the internet. There are thousands of these sensors within a manufacturing environment, from the temperature gauges to the individual components on the material handling system, connecting data from these devices can improve business efficiency, innovation as well as strengthening security. However; if the data is not connected to a single common platform talking the same language, these potential gains are lost. The aim of this research is to develop an agent based feedback control system and implement it utilizing IoT to control the material handling system in manufacturing industry.
\end{abstract}

Keywords: control system, agent based controller, material handling system, internet of things, modern manufacturing
Volume 4 Issue 4 - 2018

Aydin Azizi,' Poorya Ghafoorpoor Yazdi, ${ }^{2}$ Ali Al Humairi, ${ }^{3}$ Mohammad Alsalmi, ${ }^{4}$ Basma Al Rashdi,' Zuwaina Al Zakwani,' Sultan ALSheikaili'

'Department of Engineering, German University of Technology, Oman

${ }^{2}$ Department of Mechanical Engineering, Eastern Mediterranean University, Northern Cyprus

${ }^{3}$ Department of Computer Science, German University of Technology, Oman

${ }^{4}$ German University of Technology, Oman

Correspondence: Aydin Azizi, Engineering Department, German University of Technology, Muscat, Oman, Email aydin.azizi@gutech.edu.om

Received: May 15, 2018 | Published: July 24, 2018

\section{Introduction}

Nowadays, there has been industrial revolutions in dynamic industry. ${ }^{1}$ Products, markets, technologies and regulatory requirement have to be adapted in modern manufacturing. ${ }^{2,3}$ One of the important gadgets of modern manufacturing which has been evolved with advances in technology is Material Handling System (MHS). ${ }^{4}$ Thus, manufactures have been seeking to design intelligent MHS constructed in a way that products, work stations and system are emerged by improving the communication between units in the system to create more adaptable control of assembly flow and system performance. These communication are promoted by the aid of autonomy. ${ }^{5}$ Nowadays Design a smart MHS for manufacturing purposes becomes more complex due to the growth of automation level used in industry. ${ }^{6}$ Therefore, in recent decades by utilizing the Internet of Things (IoT) in modern manufacturing system to make it intelligent; agent based control architecture is mostly used to create a smart MHS. ${ }^{7}$ The agent system theories have been used to combine and develop the schedule of processing operations with total processing time of the tasks released by MHS. Julie et al. ${ }^{8}$ have been developed the algorithms for agent based material handling system by allocating and executing processes for all machines within the system. Zhang et $\mathrm{al}^{9}$ worked the method to control wide range of products. In their research, a variant- oriented manufacturing system was developed to be more applicable to meet the demands in modern manufacturing systems. Consequently logical entities such as controls, programs, communication protocols and human resources with a combination of tools, machines, computers, human workers have to be taken under consideration while designing and assembling any modern automotive manufacturing systems. In recent advances, there are series of ergonomic assist devices which are called "intelligent assist system "or "intelligent assist devices" or "collaborative robots", "holonomic manipulators". ${ }^{8}$ In this type of devices, a computer is used to improve the movement of heavy items in order to respond very precisely to the material handling system operations. ${ }^{10}$ These devices work by combining human flexibility, intelligence and skills by which robotic systems capabilities are promoted. The function of collaborative robots are to share the workspace with human coworker and assistance which integrates feedback with programming and motion guidance in semi-autonomous functions. ${ }^{11}$ Agent systems has become the key information technology for the next generation of manufacturing control. Motivated by the inability of existing manufacturing systems 
a. to deal with the evolution of products and

b. to maintain a satisfying performance outside normal operation.

This new manufacturing paradigm is supposed to overcome these deficits with the help of concepts like autonomy, cooperation, and self-similarity. ${ }^{12}$ Therefore, this research aims to design agent based control architecture for MHS to make it smart. The control agent has been designed in way that it is capable of sending signals to the devices to differentiate and distinguish the objects and transport it to the right place hence, making it a very smart material handling system. The proposed control algorithm implement by utilizing Mindstorm software and result successfully has been explored via existing MHS located in German University of Technology (GUtech).

\section{Design agent based controller for MHS}

\section{Overview}

The history of the industries all started with the mechanization and the use of steam to power those machines that changed the manufacturing industry for the first time with this advancement it allowed factories to set up mass production and assembly lines, it was also at this time that electricity was added to the equation, over the years along with technological advancement, computers and automated machines were created to take the work load of the assembly lines. ${ }^{13}$ Nowadays technology has grown wider, most industries need material handling system however different requirements are needed. All material handling system needs control architecture to be smart or automated. Step above control architecture agent based architecture is utilized to improve the control system. Agent technology, in this particular situation, becomes a right candidate to take on the new challenge. An agent is an active object which possesses certain capabilities to perform tasks, and it communicates with other agents based on the organizational structure to cooperate the accomplishment of tasks. ${ }^{14}$ Agent based system is a guide to a construct a software system with the aid of many existing agent's platforms. Within AgentBased Modeling (ABM) a framework is exhibited as a collection of independent decision-making agents. Each agent examines the situation individually and makes certain decisions based on set of rules. Agents can perform different behaviors which is suitable for its correspondence system (Figure $1 \& 2$ ).

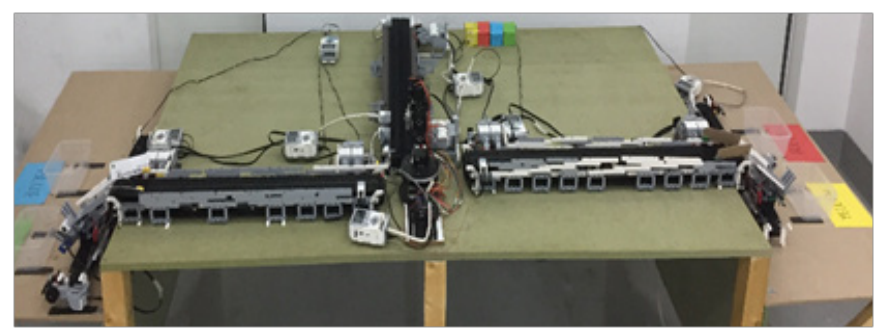

Figure I MHS located in GUtech.

\section{Architecture of agent based control system}

The basic concept of agent based is the allocation process by considering the information that matches with all system entities. ${ }^{10}$ All entities structure of software have different types of internal evaluations. ${ }^{15}$ Thus, allocation decisions which corresponds to the specific function are made based on these embedded entities structure by the aid of the agent-based architecture. ${ }^{16}$ However, the functionality of material handling entities in agent- based architecture is shown in Figure 3. There are three fundamental levels which are Constructs system level schedule, System decision making units and change existing schedules. Constructs system level schedule is responsible for global view agent while System decision making units are responsible for order agents. Furthermore, level three at the bottom is responsible for material handling agents. These three levels are integrated precisely with system monitoring and database. Global view agent is the head of this process. Its task is to communicate only with order agents. Then, order agents communicate with material handling agents. Material handling system is very significant in many environmental aspects due to its functionality in manufacturing process. Assembling material handling system is really essential for many different industries. Statics show that automotive systems in manufacturing processes can recover vast costs to the companies. ${ }^{18}$ This briefly can be achieved by improving the combination of assembly technology and system.

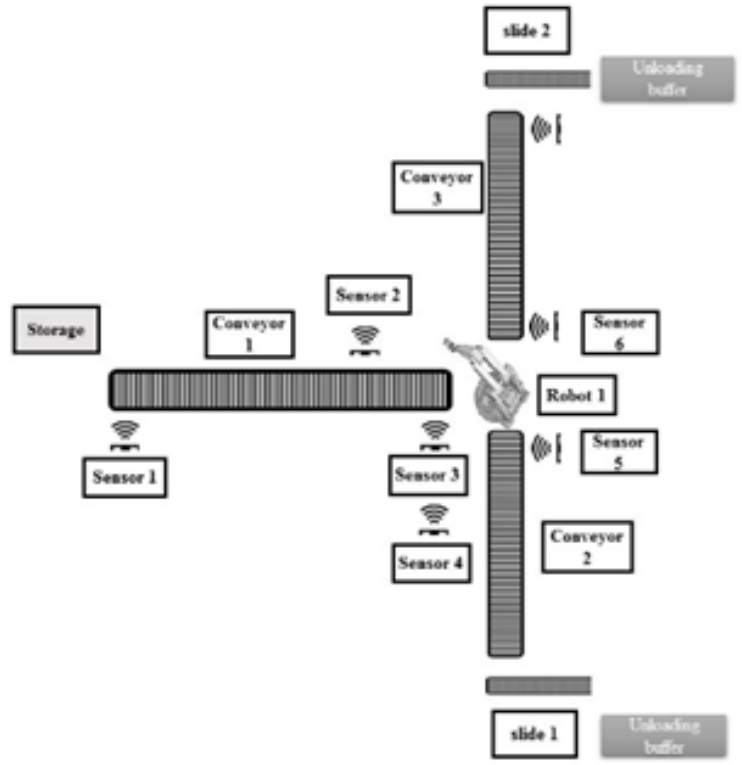

Figure 2 schematic diagram of the MHS located in GUtech. ${ }^{7}$

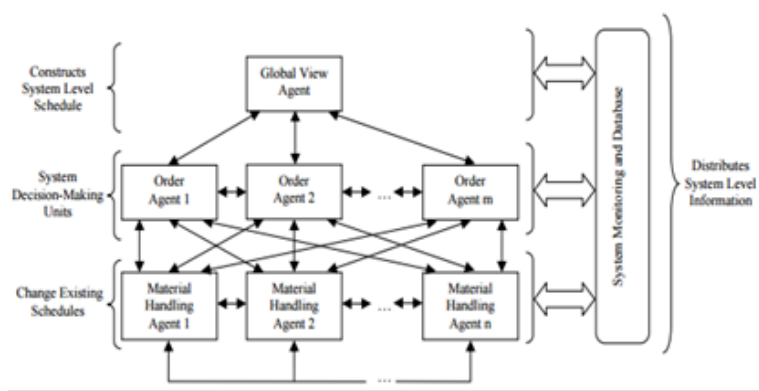

Figure 3 Agent based material Handling System Architecture. ${ }^{17}$

\section{Proposed agent based control Algorithm for the MHS}

To implement the concept of internet of things into this MHS needs a principle device that synchronizes the communication throughout the network, this device is called master which is capable of controlling all the other control agents which are called slaves. ${ }^{19}$ This can be done by using the IoT common protocol which is the Bluetooth. The master has the ability to control up to 7 slaves. Each slave will be connected to the different equipment in the system. The 
master can send and receive commands from the slaves by using the Bluetooth. The control architecture which has been proposed in this research consists of one master and 6 slaves. Figure 4, shows the proposed functionality algorithms of the main system, it includes all the hardware of the material handling system. It first starts with the main conveyor then the object it picked by the robot and according to its color feature will either be transported to conveyor 2 and then head to slider 1 or transported to conveyor 3 and head to slider 2. Each hardware will have its own proposed functionality algorithms so that it could be easily implemented into the software.

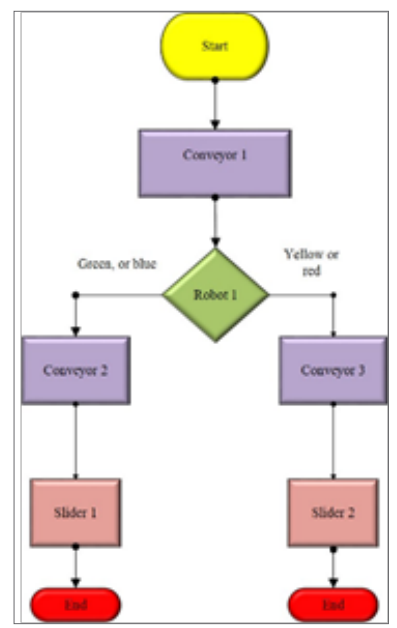

Figure 4 Proposed algorithm for the main system.

Proposed agent based control algorithm for the conveyor 1: The proposed algorithm for the main conveyor control agent summarized in Figure 5. If sensor 1 have detected the object it will activate the conveyor and if nothing is detected the conveyor will remain deactivated. As the conveyor has been activated it will move till it reaches the second sensor which once detected will lower the motion of the conveyor and continue to the last sensor which is sensor 3 , once this sensor is activated it will stop the motion of the conveyor and a Bluetooth message will be sent to the master and the master will send a signal to the robot in order to pick the object after the robot picks up the object the master will send a message to the conveyor to start functioning again. When an object is being placed in front of sensor 1 and sensor 3 at the same time the motors will still be deactivated until it receives a message back from the master.

Proposed agent based control algorithm for robot agent: The Figure 6, shows the proposed functionality for robot control agent in material handling system. It first begins by receiving a message from the master. When sensor 4 detects the object's color blue or green, the robot will pick up the object and drop it on conveyor 2 and the robot will then return to its initial position and send a message to the master. If sensor 4 detects the color red or yellow, the robot will pick up the object and drop it on conveyor 3 and the robot will then return to its initial position and send a message to the master.

Proposed agent based control algorithm for conveyors 2\&3: Figure 7, shows the proposed algorithm for conveyors 2 and 3 control agents. It has one sensor which is sensor 5 for conveyor 2 and sensor 6 for conveyor 3 when it detects an object it will handle that object until it reaches the slider 1 in conveyor 2 and slider 2 in conveyor 2 .

Proposed agent based control algorithm for slider 1: Figure 8 shows the proposed algorithm for slider 1 control agent, when sensor
7 detects the color of the object to be blue it will move to pocket 1 and drop the object and return to its initial position. If sensor 7 detects the color of the object to be green it will move to pocket 2 and drop the object and return to its initial position.

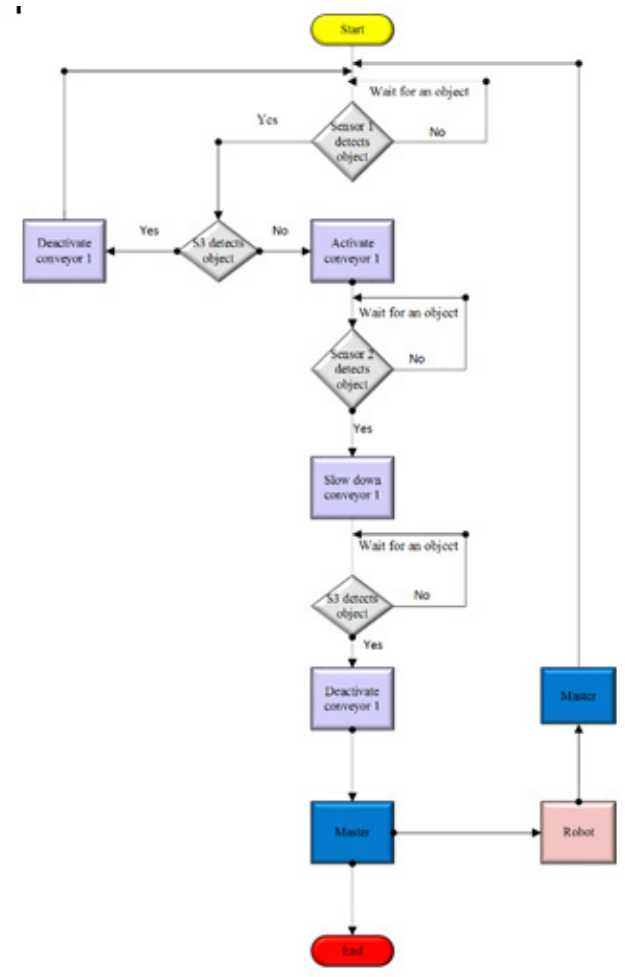

Figure 5 Proposed algorithm for the conveyor I.

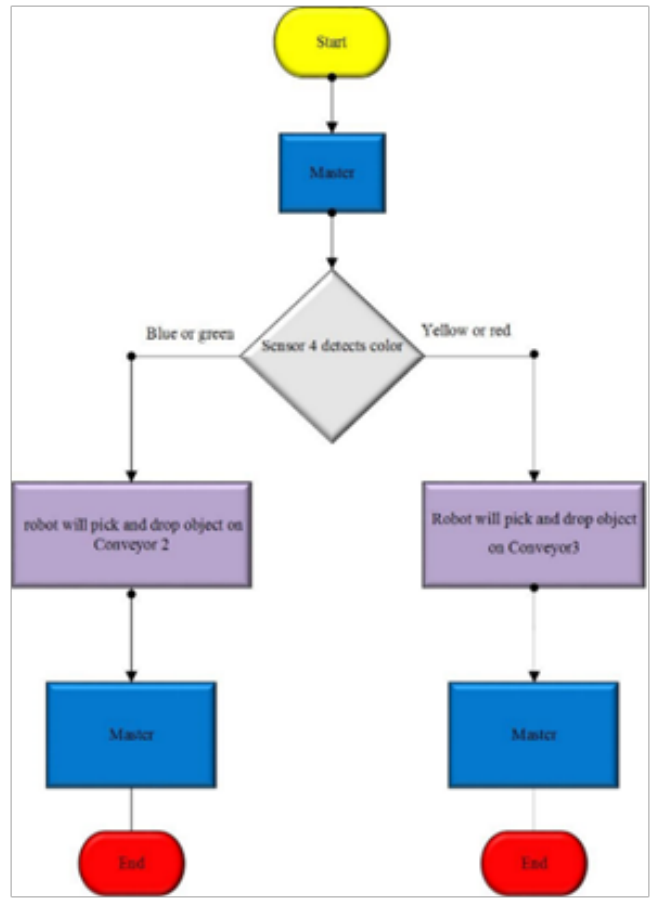

Figure 6 Proposed algorithm for the robot agent. 


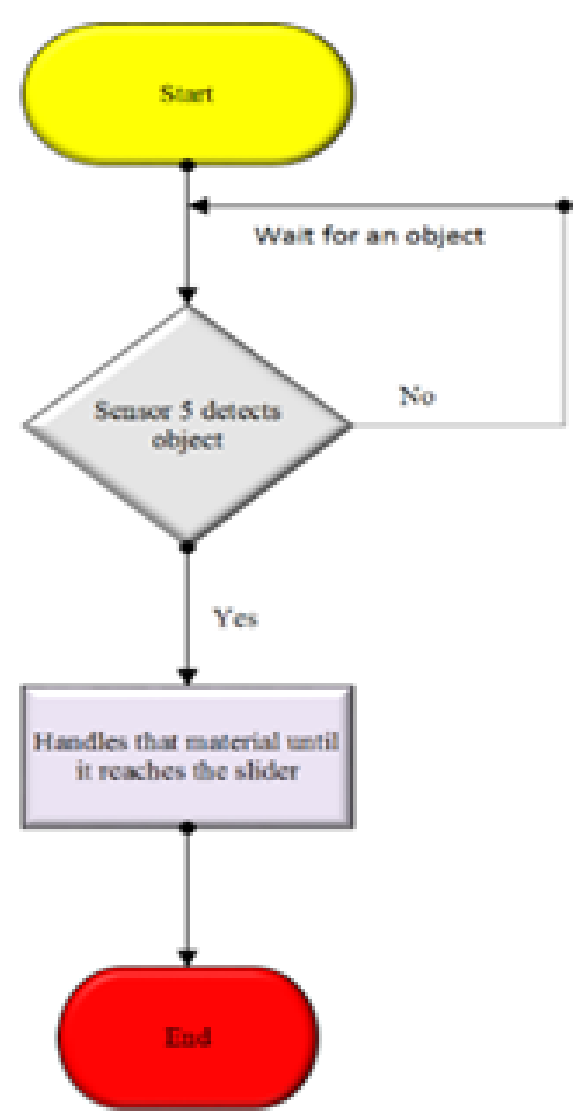

Figure 7 Proposed algorithm for conveyors $2 \& 3$.

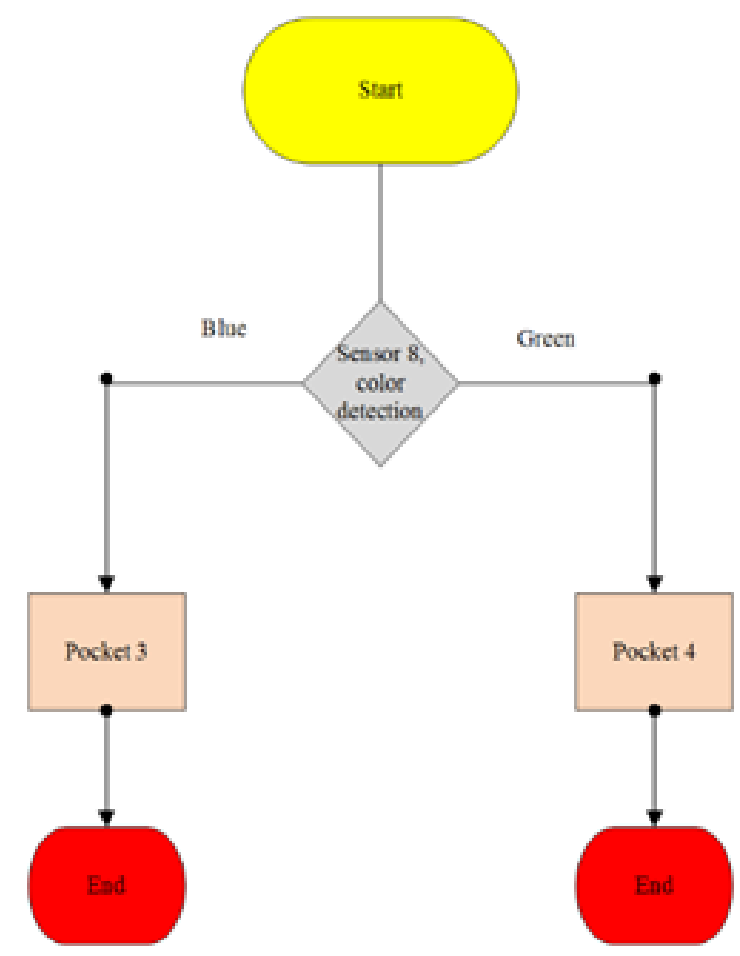

Figure 8 Proposed algorithm for sliders.

\section{Results and discussion}

The system used agents to have a centralized system. The Centralized system is controlled by the single master EV3 brick as shown in Figure 9, which has the ability to control the operation of all the slaves' bricks in the system. The master brick has the role of decision making, and all slave agents used in the framework depend on that. The slave's control agent location in the material handling system is shown in Figure 9.

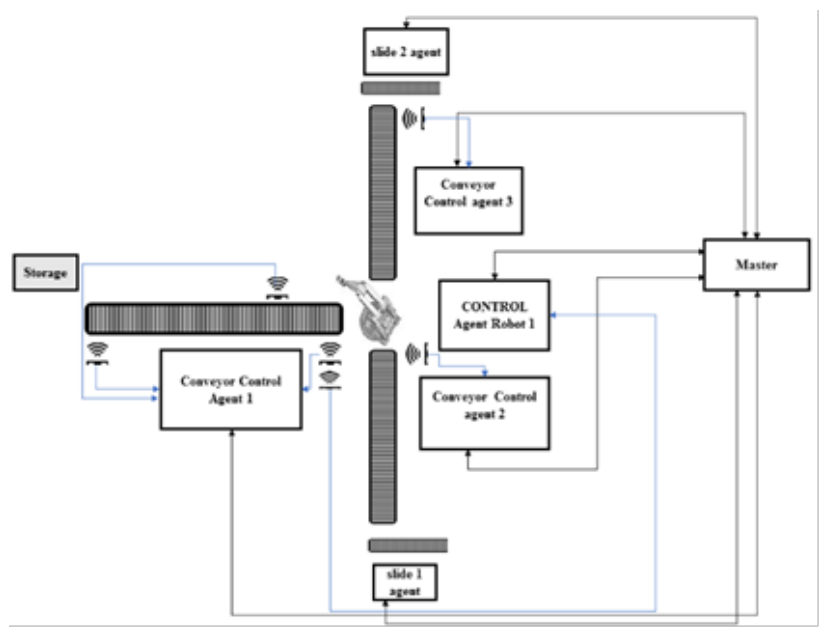

Figure 9 The main proposed agent based control system.

The proposed algorithms for all of the parts of MHS

(Conveyors, Robot arm, and Sliders) have been tested on the existing MHS located in GUtech by aid of utilizing EV3MINDSTORMS block programming software. After programming all the proposed algorithms, it resulted to a successful communication that makes the material handling system smart. The robot arm acts very smart as it only starts working when it receives a signal from the master and it can also distinguish colors by the help of sensors and distribute the objects in the right place. The results show that the proposed algorithm working properly and capable of control both master and slave parts by establishing commutation between them.

\section{Conclusion and future work}

The main purpose of this research project is to obtain a complete smart system. It starts with obtaining the software and programming it using the proposed algorithms and then implements it to the hardware components such as, conveyors, sliders and robot arm. Everything was arranged firstly from the main conveyor then transporting the material to another two conveyor belts with the aid of robot arm. The accuracy of the sensors as well as the robot arm was highly improved, after changing the positioning of both sensors and robot arm to meet the exact position that can detect object and offset the robot arm. The theory behind internet of things has been accomplished in this project, the devices can communicate with each other, and the hardware and the software are interacting to serve the purpose of internet of things. The equipment's are all connected and waits for a software signal to start a smart system. The future work could be to implement new internet of things protocols which is other than Bluetooth, nowadays there are many new protocols that fits today's generation which makes the connectivity of internet of things a much simpler task. New protocol like IoTivity, which can help transfer large data, making the system 
a bigger and smarter system. Trying different software program and utilizing Artificial Intelligent techniques ${ }^{20,21}$ can also enhance the efficiency of the system. The system in this research is a centralized system because it is controlled by master and slaves control agents, therefore in future it is recommended to have a distributed system, were the control agents can perform tasks as others, for instant if one of the control units fail to do its job, the other control units can back up and take its job, this will result to a better and much effective production line for the industry.

\section{Acknowledgements}

None.

\section{Conflict of interest}

The author declares there is no conflict of interest.

\section{References}

1. Azizi A, Vatankhah Barenji A, Hashmipour M. Optimizing radio frequency identification network planning through ring probabilistic logic neurons. Advances in Mechanical Engineering. 2016;8(8).

2. Azizi A. Introducing a novel hybrid artificial intelligence algorithm to optimize network of industrial applications in modern manufacturing. Complexity. 2017;2017:1-18.

3. Azizi A, Barenji A, Barenji R, et al. Modeling mechanical properties of fsw thick pure copper plates and optimizing it utilizing artificial intelligence techniques. Sensor Netw Data Commun. 2016;5(142):2.

4. Leitão P, Barata J. An agent-based disturbance handling architecture in manufacturing control. IFAC Proceedings Volumes. 2007:40(3):50-55.

5. Gambao E, Hernando M, Surdilovic D. A new generation of collaborative robots for material handling. Gerontechnology. 2012;11(2):368.

6. Culler D, Long J. A prototype smart materials warehouse application implemented using custom mobile robots and open source vision technology developed using emgucv. Procedia Manufacturing. 2016;5:1092-1106

7. Azizi A, Yazdi P, Humairi A. Design and fabrication of intelligent material handling system in modern manufacturing with industry 4.0 approaches. Int Rob Auto J. 2018;4(3):186-195.

8. Caricato P, Grieco A. Using simulated annealing to design a materialhandling system. IEEE intelligent systems. 2005;20(4):26-30.
9. Zhang Y, Zhang G, Du W, et al. An optimization method for shopfloor material handling based on real-time and multi-source manufacturing data. International Journal of Production Economics. 2015;165:282-292.

10. Beschorner A, Glüer D. MaxFlow theory for availability calculation of automated material handling systems. Robotics and Computer-Integrated Manufacturing. 2003;19(1):141-145.

11. Wang FK, Lin JT. Performance evaluation of an automated material handling system for a wafer fab. Robotics and Computer-Integrated Manufacturing. 2004;20(2):91-100.

12. Jennings N, Wooldridge MJ. Agent technology: foundations, applications, and markets. Springer Science \& Business Media; 1998.

13. Jeong WS, Kim SH, Min KS. An analysis of the economic effects for the IoT industry. Journal of Internet Computing and Services. 2013;14(5):119-128.

14. Birukou A, Blanzieri E, Giorgini P. Implicit: a multi-agent recommendation system for web search. Autonomous Agents and Multi-Agent Systems. 2012;24(1):141-174.

15. Compton RE. Robotic material handling applications in a flexible manufacturing system. Robotics. 1986; 2(3):259-265.

16. Lee GH. Design of components and manufacturing system for material handling in CIM. International Journal of Computer Integrated Manufacturing. 1999;12(1):39-53.

17. Beamon BM, Chen VC. Performability-based fleet sizing in a material handling system. The International Journal of Advanced Manufacturing Technology. 1998;14(6):441-449.

18. Halim NHA, Yusuf N, Jaafar R, et al. Effective material handling system for jit automotive production line. Procedia Manufacturing. $2015 ; 2: 251-257$

19. Shan WC, Lin LX, Li G, et al. A seamless operation mode transition control strategy for a microgrid based on master-slave control. In $31^{\text {st }}$ Chinese Control Conference; IEEE; 2012. p. 6768-6775.

20. Azizi A. Designing of artificial intelligence model-free controller based on output error to control wound healing process. Biosens J. 2017;6(147):2.

21. Koopialipoor M, Fallah A, Armaghani DJ, et al. Three hybrid intelligent models in estimating flyrock distance resulting from blasting. Engineering with Computers. 2018:1-14. 